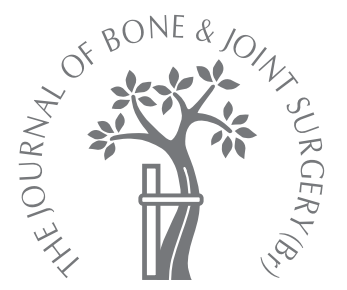

C. D. Moussallem, C. A. El-Yahchouchi, A. C. Charbel, G. Nohra

From the Lebanese University, Beirut, Lebanon

C. D. Moussallem, MD, PGY4 Orthopaedic Surgery

C. A. El-Yahchouchi, Medical Student

Faculty of Medical Sciences Lebanese University, P. O. Box 113-6155, Beirut, Lebanon.

A. C. Charbel, MD, Orthopaedic Surgeon

G. Nohra, MD, Head of Neurosurgery

Notre Dame des Secours University Hospital, PO Box 3, Byblos, Lebanon.

Correspondence should be sent to Dr C. D. Moussallem; e-mail: charbel_moussallem@ @otmail. com

(C)2009 British Editorial Society of Bone and Joint Surgery doi:10.1302/0301-620X.91B11. $22258 \$ 2.00$

$J$ Bone Joint Surg $[\mathrm{Br}]$ 2009;91-B:1531-2.

Received 31 December 2008; Accepted after revision 10 June 2009

\title{
Late spinal subdural haematoma after spinal anaesthesia for total hip replacement
}

\author{
We present a case of delayed presentation of a subdural haematoma causing cauda equina \\ syndrome which occurred 96 hours after a spinal anaesthetic had been administered for an \\ elective total hip replacement in an 86-year-old man. The patient had received low- \\ molecular-weight heparin anticoagulation which had been delayed until 12 hours post- \\ operatively. No other cause of the haemorrhage could be identified.
}

Spinal subdural haematoma is a rare but a devastating complication which followsoperation. It usually occurs shortly after the operation. We describe an 86-year-old man who developed a cauda equina syndrome due to a subdural haematoma 96 hours after total hip replacement (THR) performed under spinal anaesthesia.

\section{Case report}

An 86-year-old man with painful osteoarthritis of the left hip was advised to undergo THR. Preoperatively, other than restricted movement of the left hip, his physical and neurological examination was normal. He had no abnormal haematological investigations and his clotting parameters were within the normal range. No anticoagulants were given pre-operatively and he was not taking aspirin. Spinal anaesthesia was given by an experienced anaesthetist with the patient in the sitting position. A 25 -gauge spinal needle was successfully inserted into the L3-4 intervertebral space at the first attempt in an atraumatic manner, and $3 \mathrm{ml}$ of $0.5 \%$ hyperbaric bupivacaine were injected into the subarachnoid space. After the spinal anaesthesia the patient was positioned in the right decubitus position and the THR performed uneventfully. In the recovery room he showed normal motor function of his lower limbs. Low-molecularweight heparin (LMWH; enoxaparin, $40 \mathrm{mg}$ subcutaneously) was given once daily, starting 12 hours after surgery. On the same day after operation the patient complained of slight pain in the wound. He had no headaches or back pain and no motor or sensory deficits of the lower limbs. On the first day after operation the patient was walking with the help of a physiotherapist, and no neurological symptoms were noted. However, at 96 hours after the operation, he showed rapidly progressive sensory and motor deficits in his legs associated with mild low back pain. Neurological evaluation revealed weakness in all muscle compartments of the lower limbs. According to the grading scale of the Medical Research Council $^{1}$ muscle strength was assessed as 3 for iliopsoas, 2 for quadriceps, 2 for the hamstrings, 1 for the anterior calf muscles and 1 for the posterior calf muscles. He had overflow incontinence and a catheter was inserted. A diagnosis of cauda equina syndrome was made. MRI of the lumbar spine showed a subdural haematoma at the level of L2 and L3 with a hypointensive signal on the T2-weighted image and a moderately high signal on the T1-weighted image (Fig. 1). Compression of the nerve roots of the cauda equina caused by the formation of a haematoma was evident with a moderately narrow canal at the level of L3 and L4.

Emergency decompressive laminectomy was performed under general anaesthesia involving L2, L3 and half of the L4 lamina. The dura was distended by the underlying haematoma. A durotomy was performed and the haematoma evacuated. The arachnoid meninges were also incised to allow gentle dissection of the nerve roots and evacuation of any blood which had already reached the anterior aspect of the cauda equina. No other abnormalities such as an arteriovenous malformation or a vascular tumour were noted. During his stay in hospital the patient showed only slight improvement of his motor function and he was transferred to a rehabilitation centre. Unfortunately, one year later there was no return of his bladder control and no improvement in motor function. He is still unable to walk. 


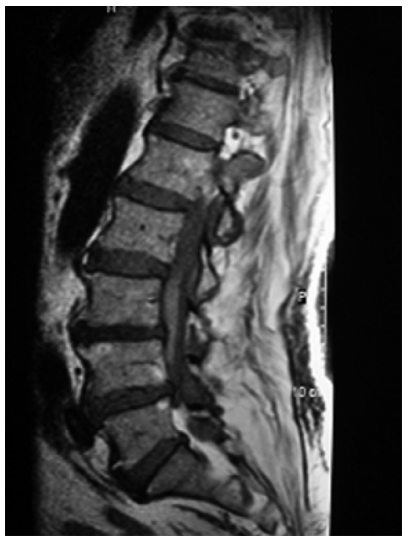

Fig. 1a

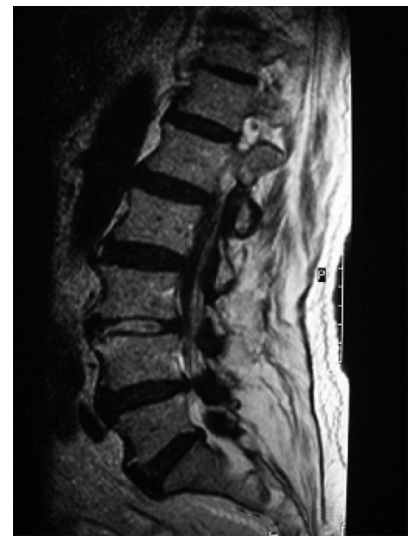

Fig. 1b
MRI showing a) a sagittal T1-weighted image with a subdural haematoma at the L2 and L3 levels and b) a T2-weighted image with a highintensity signal consistent with presence of a subdural haematoma.

\section{Discussion}

Subdural spinal haematoma is an extremely rare complication of spinal anaesthesia ${ }^{2}$ and may lead to devastating results such as a cauda equina syndrome or even permanent paraplegia. ${ }^{2-6}$ It generally occurs in patients with blood dyscrasia such as haemophilia ${ }^{7}$ or who are receiving anticoagulation therapy. ${ }^{8,9}$ Traumatic or difficult insertion of the spinal needle is a major risk factor. ${ }^{10}$ It is not clear if age by itself is an influence, but elderly patients are more prone to this complication. ${ }^{11}$ Spinal epidural haematoma is a more common complication. It occurs when inserting or even removing an epidural catheter ${ }^{12}$ and has a better prognosis if treated promptly. With subdural haematoma, severe low back pain with motor deficit and bladder dysfunction are the major clinical symptoms which usually develop shortly after the procedure at a mean of 30 hours. ${ }^{8}$ By contrast, in our patient the symptoms commenced 96 hours postoperatively. Urgent MRI must be performed immediately if this condition is suspected and any form of anticoagulation discontinued. Confirmation of the condition warrants urgent decompressive laminectomy with evacuation of the haematoma. In our patient the presence of a narrow lumbar canal may have contributed to the rapidly progressive motor dysfunction and the poor outcome.
The use of anticoagulation, especially LMWH is widespread in orthopaedic surgery, but caution should be observed when using it in the presence of a central neuroaxial block. It is recommended that LMWH should be used once daily and started 12 to 24 hours post-operatively especially, when indwelling spinal catheters are present or when concomitant antiplatelet or oral anticoagulant medication is being administered. ${ }^{13}$

Although a rare complication, medical staff should remain alert to the possibility of the formation of a haematoma when a patient presents with symptoms suggestive of compression of the spinal cord after spinal anaesthesia, especially in combination with the administration of LMWH, even several days after the procedure. Immediate intervention to decompress the vertebral canal must be undertaken to achieve the best outcome.

No benefits in any form have been or will be received from a commercial party related directly or indirectly to the subject of this article.

\section{References}

1. Medical Research Council. Aids to examination of peripheral nervous system Memorandum 45. London: Her Majesty's Stationery Office, 1976.

2. Katz J, Aidinis SJ. Complications of spinal and epidural anesthesia. J Bone Joint Surg [Am] 1980;62-A:1219-22.

3. Ozgen S, Baykan N, Dogan IV, Konya D, Pamir MN. Cauda equina syndrome after induction of spinal anaesthesia. Neurosurg Focus 2004;16:5.

4. Narouze SN, Casanova J, El-Jaberi M, Farag E, Tetzlaff JE. Inadvertent dural puncture during attempted thoracic epidural catheter placement complicated by cerbral and spinal subdural hematoma. J Clin Anesth 2006;18:132-4.

5. Edelson RN, Chernik NL, Posner JB. Spinal subdural hematomas complicating lumbar puncture. Arch Neurol 1974;31:134-7.

6. Juvonen T, Tervonen 0, Ukkola V, Klintrup HE. Widespread posttraumatic spinal subdural hematoma: imaging findings with spontaneous resolution: case report. $J$ Trauma 1994;36:262-4.

7. Eftekhar B, Ghodsi M, Ketabchi E, Bakhtiari A, Mostajabi P. Spinal subdural hematoma revealing hemophilia A in a child: a case report. BMC Blood Disord 2003;3:2.

8. Boukobza M, Haddar D, Boissonet M, Merland JJ. Spinal subdural haematoma: a study of three cases. Clin Radio/ 2001;56:475-80.

9. Moussallem CD, Helou AG, El-Yahchouchi CA, Bou Ghosn RG. Epidural catheters, anticoagulation and the risk of spinal hematoma: a review of literature. The Internet J Orthop Surg 2007:6.

10. Vandermeulen EP, Van Aken H, Vermylen J. Anticoagulants and spinal-epidural anesthesia. Anesth Analg 1994;79:1165-77.

11. Kreppel D, Antoniadis G, Sealing W. Spinal hematoma: a literature survey with meta-analysis of 613 patients. Neurosurg Rev 2003;26:1-49.

12. Varitimidis SE, Paterakis K, Dailiana ZH, Hantes M, Georgopoulou S. Epidural hematoma secondary to removal of an epidural catheter after a total knee replacement: a case report. J Bone Joint Surg [Am] 2007;89-A:2048-50.

13. Horlocker TT, Wedel DJ. Spinal and epidural blockade and perioperative low molecular weight heparin: smooth sailing on the Titanic. Anesth Analg 1998;86:11536. 